\title{
Outpatient Physicians' Satisfaction with Discharge Summaries and Perceived Need for an Electronic Discharge Summary
}

\author{
Kevin J. 0'Leary, MD \\ David M. Liebovitz, MD \\ Joseph Feinglass, PhD \\ David T. Liss \\ David W. Baker, MD, MPH
}

Division of General Internal Medicine, Feinberg School of Medicine of Northwestern University, Chicago, Illinois
Supported by a grant from the Smart Family Foundation through the Dr Steven DeAngeles Continuity of Care Fund.
BACKGROUND: Deficits in information transfer between inpatient and outpatient physicians are common and potentially dangerous.

OBJECTIVE: To evaluate satisfaction with current discharge summaries, perceptions of preventable adverse events related to suboptimal information transfer, and the perceived need for the electronic discharge summary we plan to design.

DESIGN AND PARTICIPANTS: Survey of Department of Medicine physicians with an outpatient practice.

MEASUREMENTS: Satisfaction with timeliness and quality of discharge summaries was assessed using a 5-point Likert scale. Respondents estimated the number of patients with preventable adverse events related to suboptimal information transfer at discharge.

RESULTS: Of the 416 eligible respondents, 226 completed the survey (54\%). Only $19 \%$ of the participants were satisfied or very satisfied with timeliness, and only $32 \%$ were satisfied or very satisfied with the quality of discharge summaries. Overall, $41 \%$ believed that at least 1 of their patients hospitalized in the previous 6 months had experienced a preventable adverse event related to poor transfer of information at discharge.

CONCLUSIONS: Physicians were not satisfied with the timeliness or quality of discharge summaries. Physicians indicated that suboptimal transfer of information at hospital discharge contributed to preventable adverse events. Journal of Hospital Medicine 2006;1:317-320. (C) 2006 Society of Hospital Medicine.

KEYWORDS: patient discharge, medical errors, medical record, medical informatics.

$\mathbf{T}$ welve percent of patients have been reported to have preventable or ameliorable adverse events in the period immediately following hospital discharge. ${ }^{1,2}$ A potential contributor to the number of adverse events is inadequate transfer of clinical information at hospital discharge. The discharge summary is a vital component of the transfer of information from the inpatient to the outpatient setting. Unfortunately, discharge summaries are often unavailable when follow-up care occurs and often lack important content. ${ }^{3-6}$

Many hospitals are implementing an electronic medical record systems. This creates the opportunity at hospital discharge to immediately assemble the major components of a discharge summary. With enhanced communication systems, this information can be delivered in a variety of ways with minimal delay. We report the results and evaluation of a survey of medicine faculty at an urban academic medical center about the timeliness and quality of discharge summaries, the perceived incidence of adverse 
events related to suboptimal information transfer at discharge, and the need for the electronically generated discharge summary we plan to design.

\section{METHODS}

\section{Study Site}

The study was conducted at a 753-bed academic hospital in Chicago, Illinois. Discharge summaries have traditionally been dictated by inpatient physicians and delivered to outpatient physicians by both mail and facsimile via the medical records department. The hospital has used an electronic medical record and computerized physician order entry system (PowerChart Millennium ${ }^{\circledast}$ from Cerner Corporation) since August 2004. Although all history and physicals and progress notes were documented in the electronic medical record, the system did not provide a method for delivering the discharge summaries contained in the electronic medical record to outpatient physician offices. Because of this, inpatient physicians continued to dictate discharge summaries during this study.

\section{Participants}

An advisory board consisting of 16 physicians from the Department of Medicine was convened. The advisory board gave input on needs assessment and helped to create a survey to be administered to all 425 medicine faculty who have an outpatient practice. All respondents who had at least 1 patient admitted to the hospital within the 6 months prior to the survey were eligible.

\section{Survey Content}

Our survey consisted of 2 parts. In the first part, we asked respondents to estimate how many of their patients had been discharged from the hospital in the past 6 months and to reflect on these patients as they completed the survey. Satisfaction with the timeliness and quality of discharge summaries was assessed using a 5-point Likert scale, from 5, "very satisfied," to 1 , "very unsatisfied." The frequency of hospital follow-up of a patient occurring prior to arrival of the discharge summary was assessed as the percentage of times this occurred in $20 \%$ increments $(0 \%-19 \%, 20 \%-39 \%, 40 \%-59 \%, 60 \%-79 \%$, and $80 \%-100 \%)$. The number of discharge summaries missing critical information and the number of summaries containing unnecessary information were similarly assessed using $20 \%$ increments. We then asked respondents to estimate the number of patients who had sustained a preventable adverse event related to suboptimal transfer of information at discharge. We defined a preventable adverse event as "a preventable medical problem or worsening of an existing problem."

In the second part of the survey, we elicited preferences for discharge summary content and method of delivery. We assessed preferences for discharge summary content by asking respondents to rank items on a scale from 1 to 10 , from 10, "most important," to 1, "least important." Preferences for delivery of discharge summaries were assessed by asking respondents to indicate one or more delivery methods, including facsimile, mail, the electronic medical record, and E-mail.

\section{Survey Process}

The survey was sent out in March 2005. A postcard reminder was sent out approximately 2 weeks after the initial survey was mailed. A second survey was sent to nonresponders 6 weeks after the initial survey. Simultaneously, the survey was also sent in Web-based format to nonresponders via email.

\section{Data Analysis}

Physician characteristics, including practice type, faculty appointment type, and year of medical school graduation, were provided by the hospital's medical staff office. Physician respondents and nonrespondents were compared using the chisquare test and logistic regression to determine potential response biases. We calculated means and standard deviations and percentages for categorical variables. Logistic regression was used to examine the likelihood of participants reporting any preventable adverse event related to suboptimal transfer of information. The regression model tested the likelihood of one or more preventable adverse events reported with the frequency of seeing patients for follow-up prior to the arrival of discharge summaries, controlling for participant characteristics and the number of hospitalized patients each physician had in the previous 6 months.

\section{RESULTS \\ Physician Characteristics}

The survey was sent to 425 physicians, 9 of whom were excluded because they had had no patients admitted within the past 6 months. Of the 416 eligible respondents, 2 returned a survey that was incomplete and not usable, and 226 returned a completed survey (response rate of $54 \%$ ). The characteristics of responders and nonresponders are 
TABLE 1

Participant Characteristics

\begin{tabular}{|c|c|c|c|}
\hline & $\begin{array}{l}\text { Responders } \\
(\mathrm{N}=226)\end{array}$ & $\begin{array}{l}\text { Nonresponders } \\
(\mathrm{N}=188)\end{array}$ & $P$ value \\
\hline \multicolumn{4}{|l|}{ Practice type } \\
\hline Generalist, N (\%) & $127(56.2)$ & $65(34.6)$ & \multirow[t]{2}{*}{$<.001$} \\
\hline Specialist, N (\%) & $99(43.8)$ & $123(65.4)$ & \\
\hline \multicolumn{4}{|l|}{ Faculty appointment } \\
\hline Full-time, N (\%) & $104(46.0$ & $106(56.4)$ & \multirow[t]{2}{*}{.04} \\
\hline Affiliated, N (\%) & $122(54.0)$ & $82(43.6)$ & \\
\hline \multicolumn{4}{|c|}{ Year of medical school graduation } \\
\hline Before 1990, N (\%) & $131(58.0)$ & $127(67.6)$ & \multirow[t]{2}{*}{.04} \\
\hline 1990 or later, N (\%) & $95(42.0)$ & $61(32.4)$ & \\
\hline \multicolumn{4}{|c|}{$\begin{array}{l}\text { Number of patients hospitalized } \\
\text { in last } 6 \text { months }{ }^{\mathrm{a}}\end{array}$} \\
\hline $1-4, \mathrm{~N}(\%)$ & $15(7.9)$ & & \\
\hline $5-10, \mathrm{~N}(\%)$ & $62(32.5)$ & & \\
\hline $11-19, \mathrm{~N}(\%)$ & 35 (18.3) & & \\
\hline 20 or more, $\mathrm{N}(\%)$ & 79 (41.4) & & \\
\hline
\end{tabular}

shown in Table 1. General medicine physicians completed the survey more often than specialist physicians ( $56 \%$ vs. $44 \%, P<.001$ ). Affiliated faculty were also more likely to complete the survey than full-time faculty; multivariate logistic regression revealed this was purely a function of the larger number of specialists among the full-time faculty.

\section{Timeliness and Content}

Only $19 \%$ of the participants were satisfied or very satisfied with the timeliness of discharge summaries. Among all participants, 33\% indicated that $60 \%$ or more of their patients were seen for their follow-up outpatient visit prior to the arrival of the discharge summary, and $22 \%$ indicated that for $60 \%$ or more of their patients they never received a discharge summary at all.

Only $32 \%$ of the participants were satisfied or very satisfied with the quality of discharge summaries. Among all participants, $17 \%$ believed that $60 \%$ or more of discharge summaries missed critical information. Unnecessary information in the discharge summary was less problematic; only $9 \%$ of participants indicated that $60 \%$ or more of discharge summaries contained unnecessary information.

\section{Preventable Adverse Events}

Overall, $41 \%$ of participants believed that in the previous 6 months at least one of their patients had
TABLE 2

Preferred Content of Discharge Summary Ranked by Importance

\begin{tabular}{ll}
\hline & $\begin{array}{l}\text { Mean rating } \\
\text { (scale of 1-10) }\end{array}$ \\
\hline Medications at discharge & 9.69 \\
Follow-up issues & 9.09 \\
Discharge diagnosis & 9.02 \\
List of procedures performed & 8.79 \\
Pathology reports & 8.78 \\
Pending test results & 8.68 \\
Procedure reports & 8.16 \\
Stress test reports & 8.07 \\
Dates of admission and discharge & 8.01 \\
Problem list & 7.99 \\
List of radiology tests performed & 7.84 \\
Echocardiogram reports & 7.79 \\
Follow-up appointments & 7.79 \\
Radiology reports & 7.76 \\
Names of consulting attendings & 7.64 \\
Name of inpatient attending & 7.28 \\
Labs from last hospital day & 7.08 \\
Medications at admission & 6.91 \\
Allergies & 6.56 \\
All lab results & 6.22 \\
Code status & 6.09 \\
Names of inpatient house officers & 5.64 \\
\hline
\end{tabular}

sustained a preventable adverse event related to poor transfer of information at hospital discharge. Reporting one or more preventable adverse events was positively associated with physicians' reports of how often they saw patients for a first postdischarge follow-up without having a discharge summary available. After adjusting for participant characteristics and the number of patients hospitalized by each physician, logistic regression results indicated that each $20 \%$ increase in the frequency of discharge summaries not arriving prior to patient follow-up appointments was associated with a $28 \%$ increase in the odds of a reported preventable adverse event (adjusted $\mathrm{OR}=1.28, P=.04$ ).

\section{Preferences for Content and Delivery}

The mean rating for importance of discharge summary elements is shown in Table 2. No discharge summary element had a mean rating of less than 5 . Participants preferred discharge summaries be delivered via the following methods: facsimile, $48 \%$; mail, $30 \%$; electronic medical record, $41 \%$; and Email, $30 \%$.

\section{DISCUSSION}

Our study found that outpatient physicians were not satisfied with the timeliness or the quality of 
current discharge summaries. Our findings are in agreement with previous studies demonstrating that discharge summaries were often not available to outpatient physicians ${ }^{3,4}$ and were often of poor quality. ${ }^{5,6}$

Preventable or ameliorable adverse events have been reported to occur in $12 \%$ of patients in the period immediately following hospital discharge. ${ }^{1,2}$ No studies have evaluated the relationship between discharge summaries and preventable adverse events following discharge. Our study found that $41 \%$ of outpatient physicians believed that at least one of their patients in the 6 months prior to the survey had sustained a preventable adverse event related to the suboptimal transfer of information at hospital discharge. In addition, the likelihood of physicians reporting one or more preventable adverse events increased with the frequency of seeing patients for follow-up prior to discharge summary arrival.

In preparation for revising the discharge summary, we asked outpatient physicians to rate the importance of discharge summary content and their preference for method of delivery of discharge summaries. As in previous studies, the outpatient physicians rated discharge medications, discharge diagnosis, test results, and follow-up plans as highly important. ${ }^{7,8}$ Much of this clinical data is now available in the electronic medical record. Therefore, it is possible to electronically assemble much, if not all, of discharge summary content. One recent study demonstrated that database-generated discharge summaries significantly increased the likelihood that a discharge summary was generated within 4 weeks of hospital discharge. ${ }^{9}$ The database used in that study required manual data input from a handwritten form. To our knowledge, no study has reported the experience of discharge summaries generated from an electronic medical record.

Our study had several limitations. First, our study used physician survey to assess the timeliness of receiving discharge summaries. Measuring the time to actual receipt of discharge summaries by physicians was beyond the scope of our study. Second, our study did not measure adverse events directly. Instead, we asked outpatient physicians to estimate how many of their patients discharged in the last 6 months had sustained a preventable adverse event related to suboptimal information transfer at discharge. We had limited space in the questionnaire to define the meaning of a preventable adverse event; therefore, the description in the survey does not exactly match previous definitions. ${ }^{1,2}$ Our study had a response rate of $54 \%$. It is possible that nonresponders may have been more satisfied with the quality and timeliness of discharge summaries and may have believed fewer patients experienced preventable adverse events related to suboptimal information transfer at discharge.

The results of our study suggest that the use of systems to improve the quality and delivery of discharge summaries has the potential to improve outpatient physician satisfaction and to reduce the number of preventable adverse events that occur during the vulnerable period following hospital discharge. With the use of electronic medical records, we now have the potential to automate the process of assembling and delivering clinical information with minimal delay. We are now using the information from this study to design a partially automated, high-quality discharge summary that can be delivered to outpatient physicians immediately on discharge.

Address for correspondence and reprint requests: Kevin J. O'Leary, MD, Associate Division Chief for Hospital Medicine, Division of General Internal Medicine, 675 North St. Clair, Suite 18-200, Chicago, IL 60611; Fax: (312) 695-2857; E-mail: koleary@nmff.org

Received 19 January 2006; revision received 12 May 2006; accepted 11 June 2006.

\section{REFERENCES}

1. Forster AJ, Clark HD, Menard A, et al. Adverse events among medical patient after hospital discharge. CMAJ. 2004;170: 345-349.

2. Forster AJ, Harvey JF, Peterson JF, Gandhi TK, Bates DW. The incidence and severity of adverse events affecting patients after discharge from the hospital. Ann Intern Med. 2003;138:161-167.

3. van Walraven C, Seth R, Laupacis A. Dissemination of discharge summaries. Not reaching follow-up physicians. Can Fam Physician. 2002;48:737-742.

4. van Walraven C, Seth R, Austin PC, Laupacis A. Effect of discharge summary availability during post-discharge visits on hospital readmission. J Gen Intern Med. 2002;17:186-192.

5. Wilson S, Warwick R, Chapman M, Miller R. General practitioner-hospital communications: a review of discharge summaries. J Qual Clin Pract. 2001;21:104-108.

6. Bertrand D, Rancois P, Bosson JL, Fauconnier J, Weil G. Quality assessment of discharge letters in a French university hospital. Int J Health Care Qual Assur. 1998;11:90-95.

7. Solomon JK, Maxwell RB, Hopkins AP. Content of a discharge summary from a medical ward: views of general practitioners and hospital doctors. J R Coll Physicians Lond. 1995;29:307-310.

8. van Walraven C, Rokosh E. What is necessary for highquality discharge summaries? Am J Med Qual. 1999;14:160169.

9. van Walraven C, Laupacis A, Seth R, Wells G. Dictated versus database-generated discharge summaries: a randomized clinical trial. CMAJ. 1999;160:319-326. 